\title{
A Statistical Shape Model for the Liver
}

\author{
Hans Lamecker ${ }^{1}$, Thomas Lange ${ }^{2}$, and Martin Seebass ${ }^{1}$ \\ ${ }^{1}$ Konrad-Zuse-Zentrum Berlin, Germany \\ ${ }^{2}$ Charité Berlin, Germany \\ \{lamecker@zib.de,thomas.lange\}@charite.de, seebass@zib.de
}

\begin{abstract}
The use of statistical shape models is a promising approach for robust segmentation of medical images. One of the major challenges in building a 3D shape model from a training set of segmented instances of an object is the determination of the correspondence between them. We propose a novel geometric approach that is based on minimizing the distortion of the mapping between two surfaces. In this work we investigate the accuracy and completeness of a 3D statistical shape model for the liver built from 20 manually segmented individual CT data sets. The quality of the shape model is crucial for its application as a segmentation tool.
\end{abstract}

\section{Introduction}

The variable functional anatomy [1] of the liver requires individual pre-operative planning for hepatic surgery. The segmentation of the liver is essential for this process.

A model-based approach is promising for fast, automated and robust 3D-image segmentation [2], because it uses global shape constraints for approximating the object contour even where it is not clearly defined in the image data. Some attempts have been made to extend the original $2 \mathrm{D}$-technique to $3 \mathrm{D}$. The main obstacle is the lack of a suitable 3D shape comparison, due to the difficulty of defining an optimality criterion for a good correspondence. Once criteria have been established efficient optimization schemes have to be devised.

Fleute et al. [3] establish correspondence by elastic registration of a template shape with all other shapes based on minimizing euclidean distance.

In [4] the correspondences are defined using an information theoretic minimal description length approach. Here the issue of optimality is addressed by optimizing the compactness of the model. The method has been applied to 2D-examples and an extension to 3D shapes with sphere-like topology is outlined.

In [5] the shapes are represented by their expansion into a series of elliptical harmonics. The principal component analysis is performed on the parametric description in contrast to the point distribution model used in [2]. Only shapes with sphere-like topology can be treated.

A method for 3D shape correspondence using local geometry and geodesics is described in [6] without its application to statistical shape models. It is evaluated computing residual surface distances.

Thompson et al. [7] compute a mapping from each shape onto a sphere using a deformable model approach. Correspondence between two spheres, constrained by matched anatomical feature lines, is established by means of a warping algorithm. 
In this work we describe a shape model for the liver using a novel approach for computing the surface correspondence, which easily extends to arbitrary topology. We investigate the compactness and completeness of a liver model with an application for segmentation in mind.

\section{Building a Statistical Shape Model}

In order to build a statistical shape model a set of segmentations of the shape of interest is required. From these segmentations we reconstruct triangulated surfaces for representing shape. For shape comparison and statistical analysis finding a good correspondence between the surfaces is crucial. Using the correspondence a principal component analysis of the shapes can be performed.

\subsection{Extraction of 3D-Shapes from CT-Data}

The shape of interest has to be labelled (segmented) in all CT-images in the training set. Due to a lack of fully automated methods manual and semi-automatic approaches (live wire, region growing, etc.) are used. With the Amira Visualization and Modelling Software [8] a typical segmentation of a liver in CT images (5mm slice thickness) takes about 45 minutes.

Applying the marching cubes method to the labelled images yields a triangulated surface with a stepped structure (see Fig. 1, left) due to the anisotropy of the voxels. To obtain a superior representation of the true anatomical 3D-shape we interpolate the labelling by inserting two intermediate slices between each two consecutive slices of the original image stack. A variational implicit function approach [9] produces smooth results (cf. fig. 1, right).
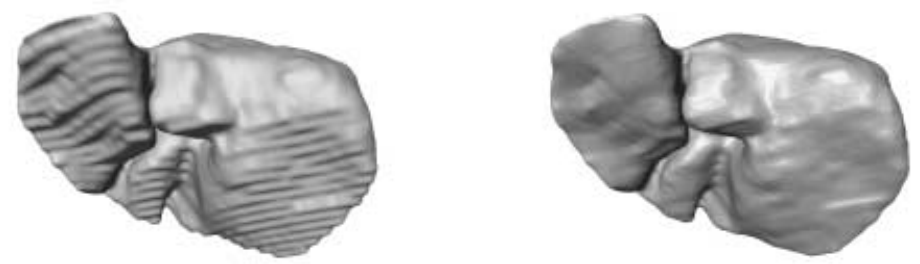

Fig. 1. Triangulated surface of the liver: before (left) and after interpolation (right)

For reasons of efficiency the surface is simplified [10] by reducing the number of triangles. A typical surface-mesh of the liver consists of about 25000 triangles and 12500 nodes.

\subsection{Solving the 3D-Correspondence Problem}

A correspondence between two 3D-shapes is a one-to-one mapping between the points on both surfaces. We propose a novel geometric approach that is based on minimizing the distortion of the mapping given a few user-defined feature points.

The method extends a morphing algorithm presented in [11]. The user defines the feature points by decomposing the surface into patches, each topologically equivalent 
to a disk. The decomposition need not only be topologically equivalent on all patches but also represent similar semantic regions in order to get a meaningful correspondence.

The patch boundaries are constructed by specifying only a few points on the surface and then computing the shortest path between them. We introduced a metric on the surface that favors paths along lines of high curvature in order to define characteristic contours (cf. fig. 2, left).

Our goal is to map any patch of one surface onto the corresponding patch on another surface minimizing the local distortion (a related idea is pursued in [7]). We define distortion as local scaling and shearing. Each patch is mapped onto a disk by applying Floater's [12] shape-preserving mapping (cf. fig. 2). This mapping approximates the geodesic polar mapping locally, which preserves arc-length in each radial direction and hence minimizes distortion. The coordinates of the parameterized points on the disk are computed by solving a sparse linear system of equations. Two corresponding disks give a one-to-one mapping from one patch to another [11], resulting in transferring one triangulation onto the other surface. To achieve continuity across patch borders, border points are mapped according to their arc-length in the particular patch of the original surface.
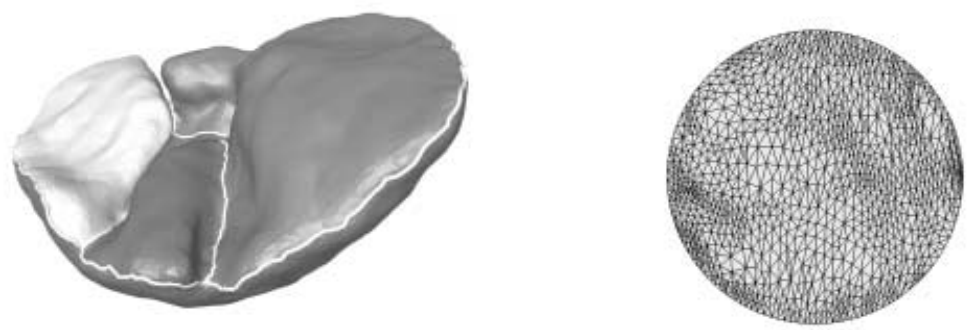

Fig. 2. Surface decomposition into patches along lines of high curvature (left) and one parametrized patch (right)

In our example, the surface of the liver is divided into four patches. The manual interaction time for the patchification is about 5 minutes per surface. The computation of the parametrization takes about 10 seconds.

\subsection{Registration of Surfaces and Principal Component Analysis}

We are now able to compare two 3D-shapes using the computed correspondence. In order to compute the mean of two shapes, they have to be aligned first. We use two different methods for this surface registration. One is the alignment of the centers of gravity of the shapes by a mere translation (TRA). The other is a rigid (MLS) transformation computed by a mean least squares fit of the displacements of the corresponding points.

We apply principal component analysis, which is a standard method for analysing variability over a set of training data [2], to the set of corresponding liver surfaces. 


\section{Results}

Based on 20 individual CT-datasets taken from a spiral CT with contrast enhancement we built an atlas following the procedure described in chapter 2. The CT-data had a slice thickness of $5 \mathrm{~mm}$ and were taken with different scanners. The training data comprised livers of 8 men and 12 women.

\subsection{Measuring Surface Similarity}

We visually perceive two shapes to be similar if no large areas on the surface deviate much from one another. Instead of just using purely global or local measures such as the mean surface distance (computed from the one sided Hausdorff-distance) or the maximal distance we adopt an intermediate measure of "region similarity". Two shapes are defined to be "region similar by X mm" if they deviate less than X $\mathrm{mm}$ on $90 \%$ of the surface.

\subsection{Shape Modes}

Fig. 3 shows the first three modes of the principal component analysis. The modes of the model are sorted in decreasing magnitude of their corresponding eigenvalues. In the first column, the mode corresponding to the largest eigenvalue $\lambda_{0}$ is varied between $-2 \sqrt{\lambda_{0}}$ and $+2 \sqrt{\lambda_{0}}$, in the second column the same is done for the second mode and so on. The result shows the large variability included in the liver model.

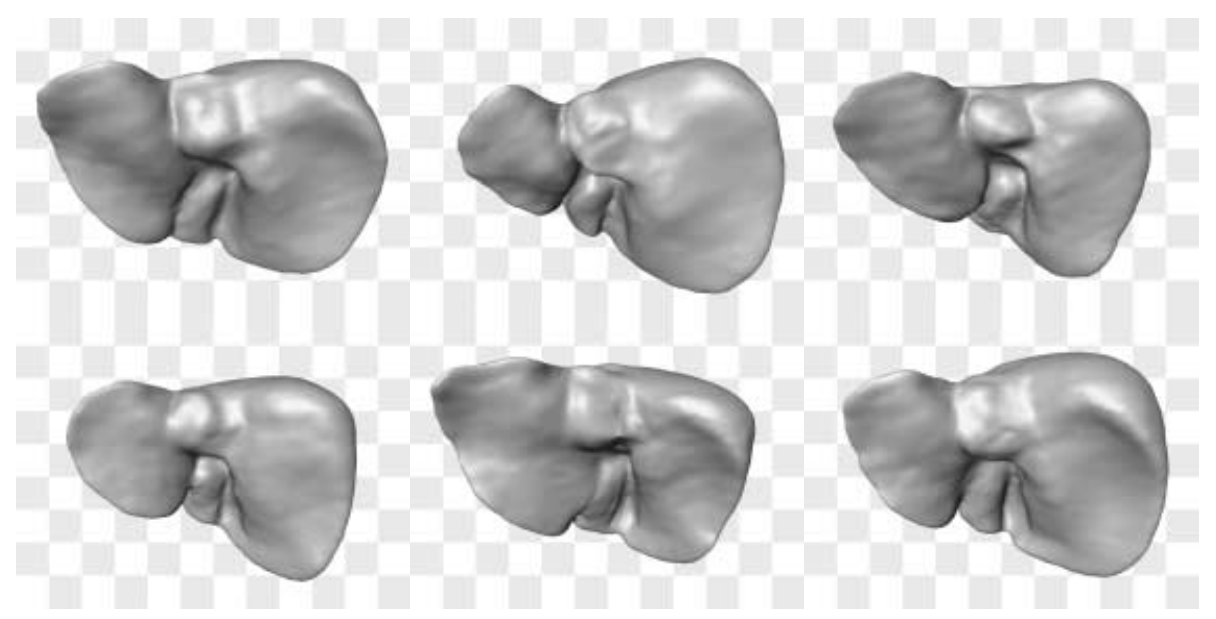

Fig. 3. First three eigenmodes of the liver atlas (columnwise)

\subsection{Compactness of the Model}

The compactness of a model is its ability to describe the variability of a shape using as few modes as possible. To figure out the compactness of our model, we first ask how much cumulative relative variance of the model needs to be taken into account when describing shapes. 
For each surface in the model we determine the number of modes, needed for creating a "region similarity by $3 \mathrm{~mm}$ " to the original one.

It turns out that on average 12 modes meet this criterion. The average distance lies in between 0.9 and $1.5 \mathrm{~mm}$, while the maximal distance ranges between 5.1 and $8.1 \mathrm{~mm}$. In fig. 4 the absolute distance between the original and the projected surface is colorcoded onto the surface.
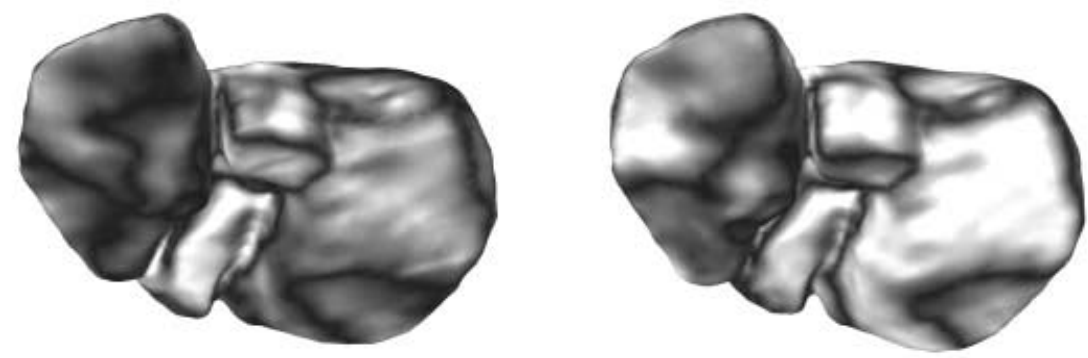

Fig. 4. Distance from original surface: 12 modes (left) vs. 10 modes (right). Black indicates a distance of $0 \mathrm{~mm}$ and white a distance of $4 \mathrm{~mm}$ and above

Fig. 5 (right) shows the cumulative relative variance plotted against the number of modes in the models, one for the TRA alignment and the other for the MLS alignment. These curves describe the compactness of our models. The curve for the MLS alignment indicates that the first 12 modes in the model contain $95 \%$ of the model's variance. Moreover we see, that the TRA model is more compact than the MLS model, while the absolute variance is larger for the TRA model. Next we determine the dimensionality of the projection space making up for more than $95 \%$ of cumulative relative variance. We plot the number of shape modes below $95 \%$ relative variance against the number of training data. For a complete model, the dimensionality should converge to a fixed value with increasing number of data in the training set. In Fig. 5 (left) convergence cannot be observed.
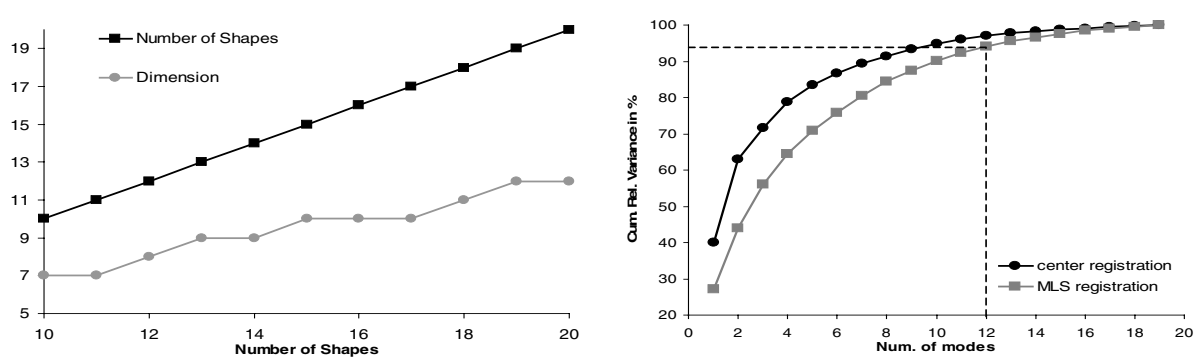

Fig. 5. Number of shapes against dimensionality (left), compactness of two models using two different alignment strategies (right)

\subsection{Representation of Arbitrary Shapes by the Model}

As a prerequisite for the application of the shape model in image segmentation we determine how accurate any shape, that is not included in the model, can be approxi- 
mated by the modes of the model. We perform a leave-one-out test on one arbitrary example surface not contained in the model to show the model's generalization capability.

The shape not contained in the model is projected onto the model using the computed correspondence. We then determine the "region similarity by $5 \mathrm{~mm}$ " between the surface and its projection. We change the threshold from $3 \mathrm{~mm}$ to $5 \mathrm{~mm}$ since we expect larger deviations than in the compactness examination. The results are shown in Fig. 6.
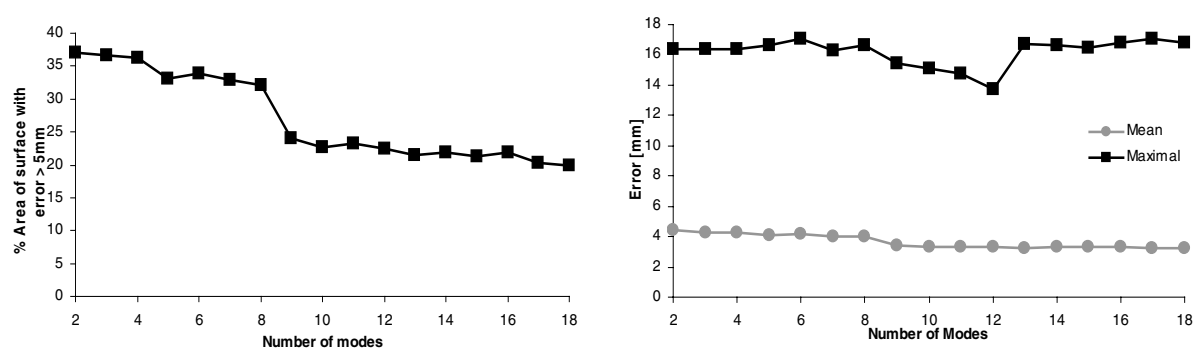

Fig. 6. Results of the leave-one-out-test: area of surface with error $>5 \mathrm{~mm}$ (left), mean and maximum surface distance (right)

\section{Discussion and Conclusion}

We have analyzed the variations of a 3D statistical shape model. One major challenge for building a shape model is the determination of a good correspondence of two surfaces. We have presented a novel method based on a patchification and the geometric idea of minimizing the distortion (local scaling and shearing of the surface). The algorithm discussed here gives only an approximate solution to this objective. As an improvement we intend to relax the surface nodes after the initial parametrization, constraining only those nodes that represent distinct features (landmarks, nodes along lines of high curvature, etc.). This relaxation should improve the correspondence and hence the representation of new shapes by the model.

The correspondence method can be extended to arbitrary topologies without difficulties. This certainly reflects one major advantage of the method presented here. Yet some more manual effort for decomposing the surface into patches is needed for more complicated topologies. We are working on an automatic extraction of anatomic feature lines.

The results of the compactness analysis show that this correspondence method is suitable for 3D shape analysis of complex and variable anatomical shape. Fig. 5 (left) indicates that the dimensionality of the model has not reached convergence. This implies that a larger training set is needed. Of course, a liver model might never be complete, though complete enough for image segmentation.

Our experiments with the leave-one-out test suggest that the essential features of an arbitrary shape will be accounted for. For the task of accurate image segmentation the model must be extended, as fig. 6 exemplifies.

Comparison between different strategies for establishing correspondence would be of high interest. As mentioned in the beginning, judging the quality of a correspondence is not trivial and may depend on the application. 
We have developed an efficient and intuitive approach for creation of 3D-shape models from segmented training data. This provides a basis for automated 3D image segmentation incorporating a-priori knowlegde.

\section{Acknowledgements}

Thomas Lange is supported by the Deutsche Forschungsgemeinschaft (DFG) project "Intraoperative Navigation" 201879. Martin Seebass and Hans Lamecker are supported by DFG collaborative research project "Hyperthermia: Clinical Aspects and Methodology" SFB 273.

\section{References}

1. Fasel, J., Selle, D., Evertsz, C., et al.: Segmental Anatomy of the Liver: Poor Correlation with CT, Radiology, 206:151-156, 1998

2. Cootes, T.,Hill, A., Taylor, C., and Haslam, J.: Use of Active Shape Models for Locating Structures in Medical Images, Image and Vision Computing, 12: 355-366, 1994

3. Fleute, M., Lavallée, S., Julliard, R.: Incorporating a Statistically Based Shape Model into a System for Computer-Assisted Anterior Cruciate Ligament Surgery, Medical Image Analysis, 3(3): 209-222, 1999

4. Davies, R., Cootes, T., Taylor, C.: A Minimum Description Length Approach To Statistal Shape Modelling, IPMI 2001, LNCS 2082: 50-63

5. Kelemen, A. , Szekely, G., Gerig, G. : Three-dimensional Model-based Segmentation of Brain MRI, IEEE Trans. on Medical Imaging, 18(10):828-839, 1999

6. Wang, Y., Peterson, B.S., Staib, L.H.: Shape-Based 3D Surface Correspondence Using Geodesics and Local Geometry, CVPR '00, 2: 644-651

7. Thompson, P.M., Toga., A.W.: Detection, Visualization and Animation of Abnormal Anatomic Structure with a Deformable Probabilistic Brain Atlas Based on Random Vector Field Transformations, Medical Image Analysis, 1(4): 271-294, 1996/97

8. Amira - Visualization and Modelling System, http://www.AmiraVis.com

9. Turk, G., O’Brian, J.F.: Shape Transformation Using Variational Implicit Functions, SIGGRAPH 99: 335-342, 1999

10. Garland, M., Heckbert, P.S.: Surface Simplification Using Quadratic Error Metrices, SIGGRAPH 97: 209-216, 1997

11. Zöckler, M., Stalling, D., Hege, H.-C.: Fast and Intuitive Generation of Geometric Shape Transitions, The Visual Computer, 16(5): 241-253, 2000

12. Floater, M.S.: Parameterization and Smooth Approximation of Surface Triangulations, Computer Aided Geometric Design, 14(3):231-250, 1997 